\title{
L-Cysteine, a Versatile Source of Sulfenic Acids. Synthesis of Enantiopure Alliin Analogues
}

Maria C. Aversa, Anna Barattucci, Paola Bonaccorsi, and Placido Giannetto

\section{Supporting Information}

Table of Contents

- X-Ray structure determination of $\left(R, E, S_{\mathrm{S}}\right)$-trimethyl-[2-(2-tert-butoxycarbonylamino2-methoxycarbonyl-ethylsulfinyl)vinyl]silane (20c) page S2 - X-ray structure of $\left(R, E, S_{\mathrm{S}}\right)$-trimethyl-[2-(2-tert-butoxycarbonylamino-2methoxycarbonyl-ethylsulfinyl)vinyl]silane (20c) (figure 1, ORTEP) page S3

- Table 1. Crystal data and structure refinement for compound 20c page S4

- Table 2. Atomic coordinates and equivalent isotropic displacement parameters for compound 20c

page 55

- Table 3. Bond lengths and angles for compound 20c page S6

- Table 4. Anisotropic displacement parameters for compound 20c page S9 
$\mathrm{X}$-Ray structure determination of $\left(R, E, S_{\mathrm{S}}\right)$-trimethyl-[2-(2-tert-butoxycarbonylamino-2methoxycarbonyl-ethylsulfinyl)vinyl]silane (20c). $\mathrm{C}_{14} \mathrm{H}_{27} \mathrm{NO}_{5} \mathrm{SSi}, \mathrm{M}=349.52$, Orthorhombic, space group P $2{ }_{1}{ }_{1}{ }_{1}{ }_{1}, a=10.159(1), b=11.094(1), c=17.907(1) \AA$, $\mathrm{V}=2018.3(2) \AA^{3}, \mathrm{Z}=4 \mathrm{D}_{\mathrm{c}}=1.150, \mu=2.162 \mathrm{~mm}^{-1}, \mathrm{~F}(000)=752$. Analysis on prismatic transparent single crystal was carried out with a Bruker CCD X-Ray diffractometer at room temperature. Crossed Gobel mirrors monochromated $\mathrm{Cu} \mathrm{K} \alpha$ radiation was used for cell parameter determination and data collection. The intensities of some equivalents were monitored during data collection to check the stability of the crystal: no loss of intensity was recognized. The integrated intensities, measured using the $\omega$ scan mode, were corrected for Lorentz and polarization effects. The reflection collected were 5641 with a $4.69<\theta<56.89^{\circ}$ range; 2571 were independent and the final $\mathrm{R}$ index was 0.0623 for reflections having $\mathrm{I}>2 \sigma \mathrm{I}$, and 0.0707 for all data. The non-hydrogen atoms were refined anisotropically; the hydrogen atoms on $\mathrm{N}_{1}, \mathrm{C}_{1}, \mathrm{C}_{2}$ and $\mathrm{C}_{2}$, were found in the Fourier difference map, while the others were assigned in calculated positions and were refined as isotropic with an overall isotropic temperature factor of $0.128 \AA^{2}$. The data acquisition, integration, data reduction and absorption correction were performed using SMART, SAINT and SADABS programs (Bruker Molecular Analysis Research Tool V 5.625, 1997-2000, Bruker AXS). This structure was solved by direct methods of SIR97 (Altomare, A.; Burla, M. C; Camalli, M.; Cascarano, G. L.; Giacovazzo, C.; Guagliardi, A.; Moliterni, A. G. G.; Polidori , G.; Spagna, R. J. Appl. Cryst. 1999, 32, 115-119) and refined using the full-matrix least squares on $\mathrm{F}^{2}$ provided by SHELXL97 (Sheldrick, G, M. SHELXL97: Program for Crystal Structure Refinement; Institut für Anorganische Chemie der Universitat Göttingen. Göttingen, Germany). Flack parameter 0.0911. Crystallographic data were deposited at Cambridge Crystallographic Data Centre, 12 Union Road, Cambridge CB2 1EZ, UK and are available from there under the deposition number CCDC 245984. 


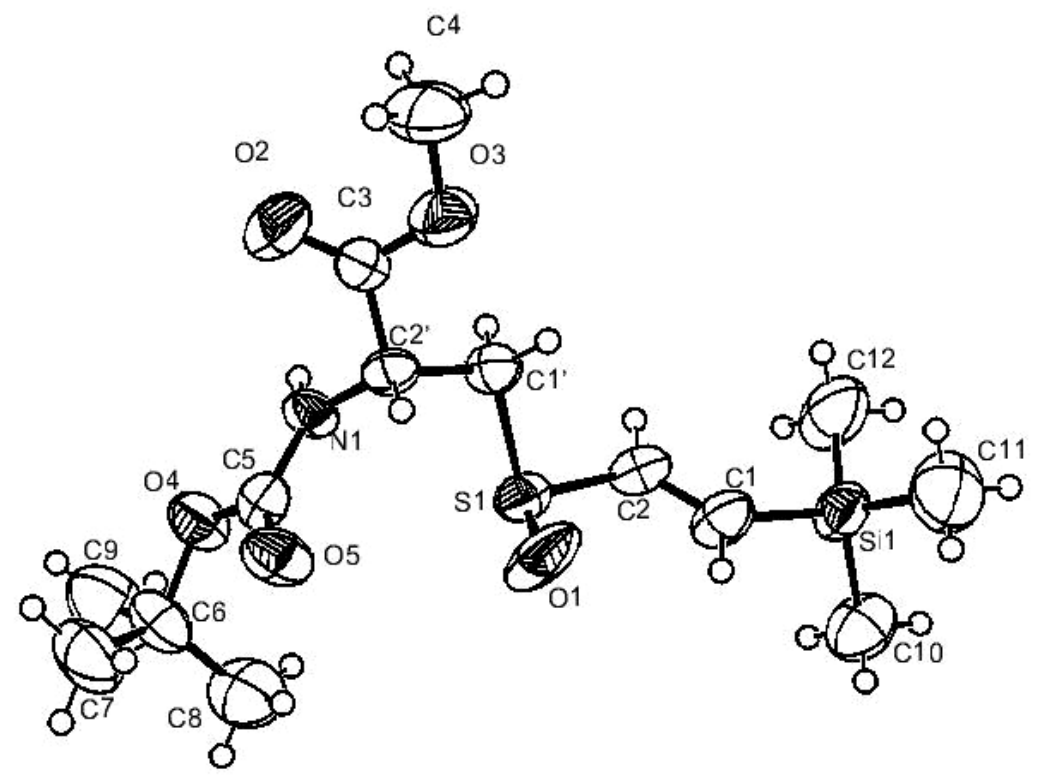

FIGURE 1. X-ray structure of $\left(R, E, S_{\mathrm{S}}\right)$-trimethyl-[2-(2-tert-butoxycarbonylamino-2methoxycarbonyl-ethylsulfinyl)vinyl]silane (20c). 
Table 1. Crystal data and structure refinement for compound $20 c$

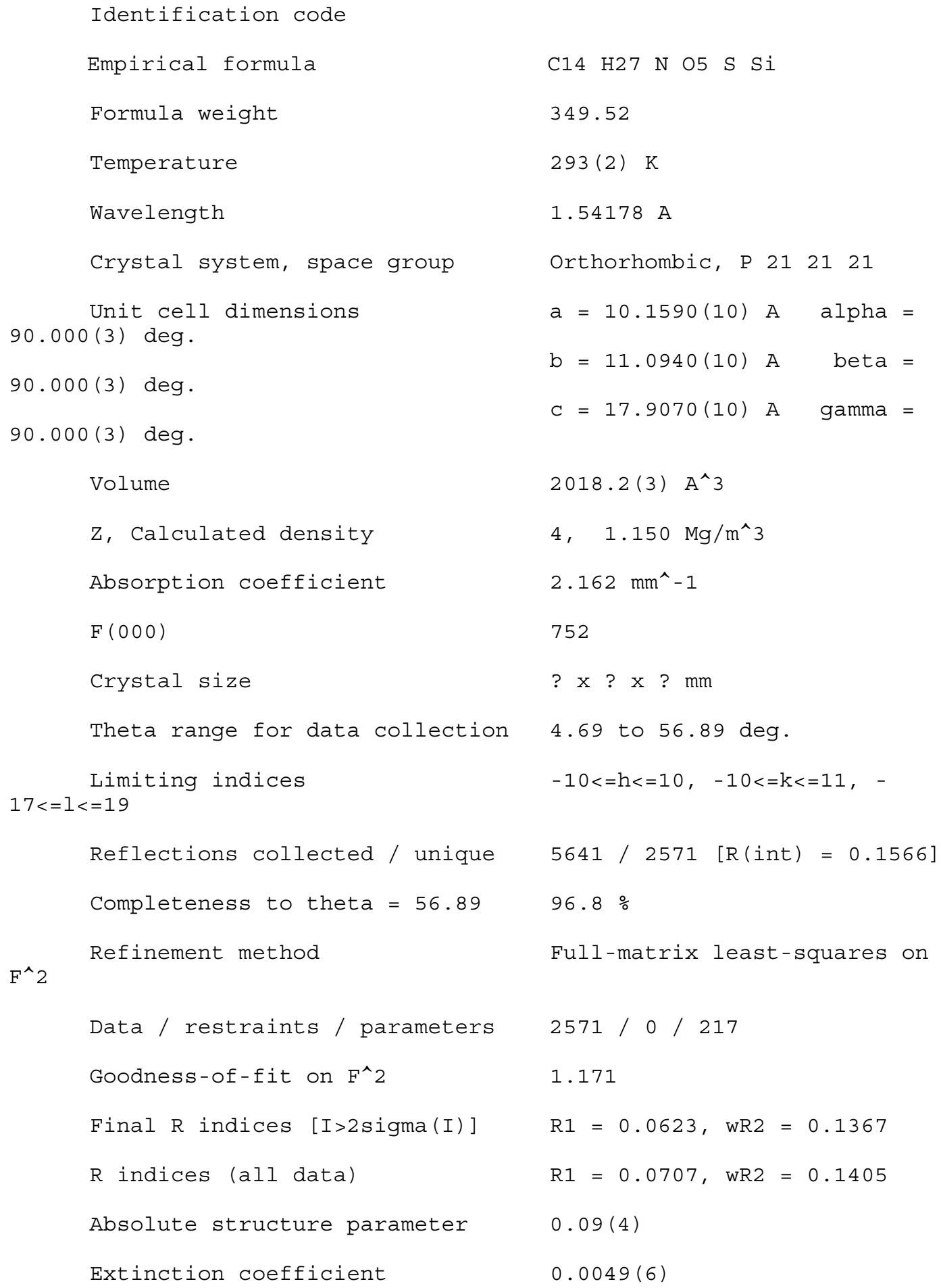


Largest diff. peak and hole

0.187 and -0.233 e.A^-3 
Table 2. Atomic coordinates ( $\left.x^{10^{\wedge}} 4\right)$ and equivalent isotropic displacement parameters $\left(A^{\wedge} 2 \times 10^{\wedge} 3\right)$ for compound $20 \mathrm{c}$ $\mathrm{U}(\mathrm{eq})$ is defined as one third of the trace of the orthogonalized

Uij tensor.

$\mathrm{U}(\mathrm{eq})$

$\mathrm{x}$

Y

z

\begin{tabular}{|c|c|c|c|c|}
\hline & $\mathrm{N}(1)$ & $-5(4)$ & $1698(4)$ & $-5801(2)$ \\
\hline 49 (1) & $O(1)$ & $-2293(4)$ & $3174(5)$ & $-4545(3)$ \\
\hline $110(2)$ & $O(2)$ & $759(5)$ & $2949(4)$ & $-7050(3)$ \\
\hline $101(2)$ & $O(3)$ & $44(4)$ & $4662(4)$ & $-6587(2)$ \\
\hline $78(1)$ & $O(4)$ & $-263(3)$ & $-241(3)$ & $-5672(3)$ \\
\hline $67(1)$ & $O(5)$ & $-2070(4)$ & $928(3)$ & $-5864(2)$ \\
\hline $68(1)$ & Si (1) & $-983(2)$ & $5670(1)$ & $-2494(1)$ \\
\hline $66(1)$ & $S(1)$ & $-879(1)$ & $3009(1)$ & $-4390(1)$ \\
\hline $62(1)$ & $C(1)$ & $-1288(6)$ & $4662(6)$ & $-3293(3)$ \\
\hline $67(2)$ & $C\left(1^{\prime}\right)$ & $-22(5)$ & $3679(4)$ & $-5176(3)$ \\
\hline $52(1)$ & $C(2)$ & $-427(6)$ & $4097(5)$ & $-3714(3)$ \\
\hline $57(1)$ & $C\left(2^{\prime}\right)$ & $-361(5)$ & $2932(5)$ & $-5875(3)$ \\
\hline $46(1)$ & $C(3)$ & $216(5)$ & $3497(5)$ & $-6572(3)$ \\
\hline $54(1)$ & $\mathrm{C}(4)$ & $454(8)$ & $5312(7)$ & $-7241(5)$ \\
\hline $107(3)$ & $C(5)$ & $-884(6)$ & $810(4)$ & $-5788(3)$ \\
\hline $51(1)$ & $C(6)$ & $-966(7)$ & $-1308(5)$ & $-5412(3)$ \\
\hline $69(2)$ & $C(7)$ & $-1904(8)$ & $-1747(6)$ & $-5999(4)$ \\
\hline $102(3)$ & $C(8)$ & $-1672(8)$ & $-1019(7)$ & $-4687(4)$ \\
\hline $101(2)$ & $C(9)$ & $115(8)$ & $-2173(6)$ & $-5268(7)$ \\
\hline $132(4)$ & $C(10)$ & $-1578(7)$ & $4916(7)$ & $-1634(4)$ \\
\hline $90(2)$ & $C(11)$ & $-1946(9)$ & $7078(7)$ & $-2641(5)$ \\
\hline $114(3)$ & $C(12)$ & $802(7)$ & $5958(8)$ & $-2406(5)$ \\
\hline
\end{tabular}


Table 3. Bond lengths [A] and angles [deg] for compound

$20 \mathrm{c}$

\begin{tabular}{|c|c|}
\hline$N(1)-C(5)$ & $1.331(6)$ \\
\hline$N(1)-C\left(2^{\prime}\right)$ & $1.422(7)$ \\
\hline $\mathrm{N}(1)-\mathrm{HN} 1$ & $0.81(6)$ \\
\hline$O(1)-S(1)$ & $1.474(5)$ \\
\hline $\mathrm{O}(2)-\mathrm{C}(3)$ & $1.185(6)$ \\
\hline$O(3)-C(3)$ & $1.305(6)$ \\
\hline$O(3)-C(4)$ & $1.437(8)$ \\
\hline $\mathrm{O}(4)-\mathrm{C}(5)$ & $1.342(6)$ \\
\hline $\mathrm{O}(4)-\mathrm{C}(6)$ & $1.459(7)$ \\
\hline $\mathrm{O}(5)-\mathrm{C}(5)$ & $1.219(6)$ \\
\hline $\mathrm{Si}(1)-\mathrm{C}(1)$ & $1.843(6)$ \\
\hline $\mathrm{Si}(1)-\mathrm{C}(12)$ & $1.848(7)$ \\
\hline $\mathrm{Si}(1)-\mathrm{C}(10)$ & $1.854(7)$ \\
\hline $\mathrm{Si}(1)-\mathrm{C}(11)$ & $1.862(8)$ \\
\hline$S(1)-C(2)$ & $1.770(6)$ \\
\hline$S(1)-C\left(1^{\prime}\right)$ & $1.814(5)$ \\
\hline$C(1)-C(2)$ & $1.314(8)$ \\
\hline $\mathrm{C}(1)-\mathrm{H}(1)$ & $0.89(5)$ \\
\hline$C\left(1^{\prime}\right)-C\left(2^{\prime}\right)$ & $1.539(7)$ \\
\hline$C\left(I^{\prime}\right)-H\left(I^{\prime} A\right)$ & 0.9700 \\
\hline$C\left(1^{\prime}\right)-H\left(I^{\prime} B\right)$ & 0.9700 \\
\hline $\mathrm{C}(2)-\mathrm{H}(2)$ & $0.90(5)$ \\
\hline$C\left(2^{\prime}\right)-C(3)$ & $1.515(7)$ \\
\hline $\mathrm{C}\left(2^{\prime}\right)-\mathrm{H}\left(2^{\prime}\right)$ & $0.92(5)$ \\
\hline $\mathrm{C}(4)-\mathrm{H}(4 \mathrm{~A})$ & 0.9600 \\
\hline $\mathrm{C}(4)-\mathrm{H}(4 \mathrm{~B})$ & 0.9600 \\
\hline $\mathrm{C}(4)-\mathrm{H}(4 \mathrm{C})$ & 0.9600 \\
\hline$C(6)-C(9)$ & $1.481(9)$ \\
\hline$C(6)-C(7)$ & $1.501(9)$ \\
\hline$C(6)-C(8)$ & $1.517(10)$ \\
\hline $\mathrm{C}(7)-\mathrm{H}(7 \mathrm{~A})$ & 0.9600 \\
\hline $\mathrm{C}(7)-\mathrm{H}(7 \mathrm{~B})$ & 0.9600 \\
\hline $\mathrm{C}(7)-\mathrm{H}(7 \mathrm{C})$ & 0.9600 \\
\hline $\mathrm{C}(8)-\mathrm{H}(8 \mathrm{~A})$ & 0.9600 \\
\hline $\mathrm{C}(8)-\mathrm{H}(8 \mathrm{~B})$ & 0.9600 \\
\hline $\mathrm{C}(8)-\mathrm{H}(8 \mathrm{C})$ & 0.9600 \\
\hline $\mathrm{C}(9)-\mathrm{H}(9 \mathrm{~A})$ & 0.9600 \\
\hline $\mathrm{C}(9)-\mathrm{H}(9 \mathrm{~B})$ & 0.9600 \\
\hline $\mathrm{C}(9)-\mathrm{H}(9 \mathrm{C})$ & 0.9600 \\
\hline $\mathrm{C}(10)-\mathrm{H}(10 \mathrm{~A})$ & 0.9600 \\
\hline $\mathrm{C}(10)-\mathrm{H}(10 \mathrm{~B})$ & 0.9600 \\
\hline $\mathrm{C}(10)-\mathrm{H}(10 \mathrm{C})$ & 0.9600 \\
\hline $\mathrm{C}(11)-\mathrm{H}(11 \mathrm{~A})$ & 0.9600 \\
\hline $\mathrm{C}(11)-\mathrm{H}(11 \mathrm{~B})$ & 0.9600 \\
\hline $\mathrm{C}(11)-\mathrm{H}(11 \mathrm{C})$ & 0.9600 \\
\hline $\mathrm{C}(12)-\mathrm{H}(12 \mathrm{~A})$ & 0.9600 \\
\hline $\mathrm{C}(12)-\mathrm{H}(12 \mathrm{~B})$ & 0.9600 \\
\hline $\mathrm{C}(12)-\mathrm{H}(12 \mathrm{C})$ & 0.9600 \\
\hline$C(5)-N(1)-C\left(2^{\prime}\right)$ & $122.9(4)$ \\
\hline $\mathrm{C}(5)-\mathrm{N}(1)-\mathrm{HN} 1$ & $126(4)$ \\
\hline $\mathrm{C}\left(2^{\prime}\right)-\mathrm{N}(1)-\mathrm{HN} 1$ & $112(4)$ \\
\hline$C(3)-O(3)-C(4)$ & $118.4(5)$ \\
\hline$C(5)-O(4)-C(6)$ & $121.6(4)$ \\
\hline
\end{tabular}




\begin{tabular}{|c|c|}
\hline$C(1)-S i(1)-C(12)$ & $109.6(3)$ \\
\hline$C(1)-S i(1)-C(10)$ & $108.4(3)$ \\
\hline$C(12)-S i(1)-C(10)$ & $109.2(4)$ \\
\hline$C(1)-S i(1)-C(11)$ & $108.1(3)$ \\
\hline$C(12)-S i(1)-C(11)$ & $112.5(4)$ \\
\hline$C(10)-S i(1)-C(11)$ & $109.0(4)$ \\
\hline$O(1)-S(1)-C(2)$ & $107.2(3)$ \\
\hline$O(1)-S(1)-C\left(1^{\prime}\right)$ & $105.7(3)$ \\
\hline$C(2)-S(1)-C\left(1^{\prime}\right)$ & $97.3(2)$ \\
\hline$C(2)-C(1)-S i(1)$ & $128.5(5)$ \\
\hline $\mathrm{C}(2)-\mathrm{C}(1)-\mathrm{H}(1)$ & $119(3)$ \\
\hline $\mathrm{Si}(1)-\mathrm{C}(1)-\mathrm{H}(1)$ & $112(3)$ \\
\hline$C\left(2^{\prime}\right)-C\left(1^{\prime}\right)-S(1)$ & $107.6(3)$ \\
\hline $\mathrm{C}\left(2^{\prime}\right)-\mathrm{C}\left(\mathrm{I}^{\prime}\right)-\mathrm{H}\left(\mathrm{I}^{\prime} \mathrm{A}\right)$ & 110.2 \\
\hline$S(1)-C\left(I^{\prime}\right)-H\left(I^{\prime} A\right)$ & 110.2 \\
\hline $\mathrm{C}\left(2^{\prime}\right)-\mathrm{C}\left(\mathrm{I}^{\prime}\right)-\mathrm{H}\left(\mathrm{I}^{\prime} \mathrm{B}\right)$ & 110.2 \\
\hline $\mathrm{S}(1)-\mathrm{C}\left(1^{\prime}\right)-\mathrm{H}\left(\mathrm{I}^{\prime} \mathrm{B}\right)$ & 110.2 \\
\hline $\mathrm{H}\left(I^{\prime} A\right)-\mathrm{C}\left(I^{\prime}\right)-\mathrm{H}\left(I^{\prime} B\right)$ & 108.5 \\
\hline$C(1)-C(2)-S(1)$ & $123.0(5)$ \\
\hline $\mathrm{C}(1)-\mathrm{C}(2)-\mathrm{H}(2)$ & $126(3)$ \\
\hline$S(1)-C(2)-H(2)$ & $111(3)$ \\
\hline$N(1)-C\left(2^{\prime}\right)-C(3)$ & $112.0(4)$ \\
\hline$N(1)-C\left(2^{\prime}\right)-C\left(1^{\prime}\right)$ & $112.7(4)$ \\
\hline$C(3)-C\left(2^{\prime}\right)-C\left(1^{\prime}\right)$ & $111.2(4)$ \\
\hline $\mathrm{N}(1)-\mathrm{C}\left(2^{\prime}\right)-\mathrm{H}\left(2^{\prime}\right)$ & $112(3)$ \\
\hline $\mathrm{C}(3)-\mathrm{C}\left(2^{\prime}\right)-\mathrm{H}\left(2^{\prime}\right)$ & $102(3)$ \\
\hline $\mathrm{C}\left(1^{\prime}\right)-\mathrm{C}\left(2^{\prime}\right)-\mathrm{H}\left(2^{\prime}\right)$ & $106(3)$ \\
\hline$O(2)-C(3)-O(3)$ & $123.7(5)$ \\
\hline $\mathrm{O}(2)-\mathrm{C}(3)-\mathrm{C}\left(2^{\prime}\right)$ & $124.3(5)$ \\
\hline$O(3)-C(3)-C\left(2^{\prime}\right)$ & $112.0(5)$ \\
\hline$O(3)-C(4)-H(4 A)$ & 109.5 \\
\hline $\mathrm{O}(3)-\mathrm{C}(4)-\mathrm{H}(4 \mathrm{~B})$ & 109.5 \\
\hline $\mathrm{H}(4 \mathrm{~A})-\mathrm{C}(4)-\mathrm{H}(4 \mathrm{~B})$ & 109.5 \\
\hline $\mathrm{O}(3)-\mathrm{C}(4)-\mathrm{H}(4 \mathrm{C})$ & 109.5 \\
\hline $\mathrm{H}(4 \mathrm{~A})-\mathrm{C}(4)-\mathrm{H}(4 \mathrm{C})$ & 109.5 \\
\hline $\mathrm{H}(4 \mathrm{~B})-\mathrm{C}(4)-\mathrm{H}(4 \mathrm{C})$ & 109.5 \\
\hline$O(5)-C(5)-N(1)$ & $125.6(5)$ \\
\hline $\mathrm{O}(5)-\mathrm{C}(5)-\mathrm{O}(4)$ & $125.1(5)$ \\
\hline $\mathrm{N}(1)-\mathrm{C}(5)-\mathrm{O}(4)$ & $109.3(5)$ \\
\hline$O(4)-C(6)-C(9)$ & $102.6(5)$ \\
\hline $\mathrm{O}(4)-\mathrm{C}(6)-\mathrm{C}(7)$ & $110.5(5)$ \\
\hline$C(9)-C(6)-C(7)$ & $112.4(6)$ \\
\hline $\mathrm{O}(4)-\mathrm{C}(6)-\mathrm{C}(8)$ & $109.5(5)$ \\
\hline$C(9)-C(6)-C(8)$ & $109.8(7)$ \\
\hline$C(7)-C(6)-C(8)$ & $111.6(6)$ \\
\hline$C(6)-C(7)-H(7 A)$ & 109.5 \\
\hline$C(6)-C(7)-H(7 B)$ & 109.5 \\
\hline $\mathrm{H}(7 \mathrm{~A})-\mathrm{C}(7)-\mathrm{H}(7 \mathrm{~B})$ & 109.5 \\
\hline $\mathrm{C}(6)-\mathrm{C}(7)-\mathrm{H}(7 \mathrm{C})$ & 109.5 \\
\hline $\mathrm{H}(7 \mathrm{~A})-\mathrm{C}(7)-\mathrm{H}(7 \mathrm{C})$ & 109.5 \\
\hline $\mathrm{H}(7 \mathrm{~B})-\mathrm{C}(7)-\mathrm{H}(7 \mathrm{C})$ & 109.5 \\
\hline$C(6)-C(8)-H(8 A)$ & 109.5 \\
\hline$C(6)-C(8)-H(8 B)$ & 109.5 \\
\hline $\mathrm{H}(8 \mathrm{~A})-\mathrm{C}(8)-\mathrm{H}(8 \mathrm{~B})$ & 109.5 \\
\hline $\mathrm{C}(6)-\mathrm{C}(8)-\mathrm{H}(8 \mathrm{C})$ & 109.5 \\
\hline $\mathrm{H}(8 \mathrm{~A})-\mathrm{C}(8)-\mathrm{H}(8 \mathrm{C})$ & 109.5 \\
\hline $\mathrm{H}(8 \mathrm{~B})-\mathrm{C}(8)-\mathrm{H}(8 \mathrm{C})$ & 109.5 \\
\hline$C(6)-C(9)-H(9 A)$ & 109.5 \\
\hline$C(6)-C(9)-H(9 B)$ & 109.5 \\
\hline $\mathrm{H}(9 \mathrm{~A})-\mathrm{C}(9)-\mathrm{H}(9 \mathrm{~B})$ & 109.5 \\
\hline
\end{tabular}




$\begin{array}{ll}\mathrm{C}(6)-\mathrm{C}(9)-\mathrm{H}(9 \mathrm{C}) & 109.5 \\ \mathrm{H}(9 \mathrm{~A})-\mathrm{C}(9)-\mathrm{H}(9 \mathrm{C}) & 109.5 \\ \mathrm{H}(9 \mathrm{~B})-\mathrm{C}(9)-\mathrm{H}(9 \mathrm{C}) & 109.5 \\ \mathrm{Si}(1)-\mathrm{C}(10)-\mathrm{H}(10 \mathrm{~A}) & 109.5 \\ \mathrm{Si}(1)-\mathrm{C}(10)-\mathrm{H}(10 \mathrm{~B}) & 109.5 \\ \mathrm{H}(10 \mathrm{~A})-\mathrm{C}(10)-\mathrm{H}(10 \mathrm{~B}) & 109.5 \\ \mathrm{Si}(1)-\mathrm{C}(10)-\mathrm{H}(10 \mathrm{C}) & 109.5 \\ \mathrm{H}(10 \mathrm{~A})-\mathrm{C}(10)-\mathrm{H}(10 \mathrm{C}) & 109.5 \\ \mathrm{H}(10 \mathrm{~B})-\mathrm{C}(10)-\mathrm{H}(10 \mathrm{C}) & 109.5 \\ \mathrm{Si}(1)-\mathrm{C}(11)-\mathrm{H}(11 \mathrm{~A}) & 109.5 \\ \mathrm{Si}(1)-\mathrm{C}(11)-\mathrm{H}(11 \mathrm{~B}) & 109.5 \\ \mathrm{H}(11 \mathrm{~A})-\mathrm{C}(11)-\mathrm{H}(11 \mathrm{~B}) & 109.5 \\ \mathrm{Si}(1)-\mathrm{C}(11)-\mathrm{H}(11 \mathrm{C}) & 109.5 \\ \mathrm{H}(11 \mathrm{~A})-\mathrm{C}(11)-\mathrm{H}(11 \mathrm{C}) & 109.5 \\ \mathrm{H}(11 \mathrm{~B})-\mathrm{C}(11)-\mathrm{H}(11 \mathrm{C}) & 109.5 \\ \mathrm{Si}(1)-\mathrm{C}(12)-\mathrm{H}(12 \mathrm{~A}) & 109.5 \\ \mathrm{Si}(1)-\mathrm{C}(12)-\mathrm{H}(12 \mathrm{~B}) & 109.5 \\ \mathrm{H}(12 \mathrm{~A})-\mathrm{C}(12)-\mathrm{H}(12 \mathrm{~B}) & 109.5 \\ \mathrm{Si}(1)-\mathrm{C}(12)-\mathrm{H}(12 \mathrm{C}) & 109.5 \\ \mathrm{H}(12 \mathrm{~A})-\mathrm{C}(12)-\mathrm{H}(12 \mathrm{C}) & 109.5 \\ \mathrm{H}(12 \mathrm{~B})-\mathrm{C}(12)-\mathrm{H}(12 \mathrm{C}) & 109.5\end{array}$


Table 4. Anisotropic displacement parameters $\left(A^{\wedge} 2 \times 10^{\wedge} 3\right)$ for compound $20 \mathrm{c}$

The anisotropic displacement factor exponent takes the form:

$-2 \mathrm{pi}^{\wedge} 2$ [ h^2 $\left.\mathrm{a}{ }^{\wedge} 2 \mathrm{U} 11+\ldots+2 \mathrm{~h} \mathrm{k} a * \mathrm{~b} * \mathrm{U} 12\right]$

\begin{tabular}{|c|c|c|c|c|c|c|}
\hline & U11 & $\mathrm{U} 22$ & U33 & U23 & U13 & $\mathrm{U} 12$ \\
\hline \multicolumn{7}{|l|}{-} \\
\hline $\mathrm{N}(1)$ & $30(3)$ & $49(3)$ & $69(3)$ & $-2(2)$ & $-2(2)$ & $-9(2)$ \\
\hline$O(1)$ & $45(3)$ & $194(6)$ & $90(3)$ & $-65(4)$ & 27 (2) & $-36(3)$ \\
\hline$O(2)$ & $142(4)$ & $91(3)$ & $70(3)$ & $-1(3)$ & $45(3)$ & $0(3)$ \\
\hline$O(3)$ & $93(3)$ & $68(3)$ & $72(3)$ & $16(2)$ & $25(2)$ & $8(2)$ \\
\hline$O(4)$ & $50(2)$ & $50(2)$ & $101(3)$ & $-3(2)$ & $-2(2)$ & $-3(2)$ \\
\hline$O(5)$ & $38(2)$ & $66(2)$ & $100(3)$ & $7(2)$ & $-9(2)$ & $-7(2)$ \\
\hline Si (1) & $67(1)$ & $78(1)$ & $53(1)$ & $-10(1)$ & $10(1)$ & $1(1)$ \\
\hline$S(1)$ & $65(1)$ & $71(1)$ & $51(1)$ & $-5(1)$ & $16(1)$ & $-15(1)$ \\
\hline$C(1)$ & $46(4)$ & $97(5)$ & $59(4)$ & $-8(3)$ & $5(3)$ & $-2(3)$ \\
\hline$C\left(1^{\prime}\right)$ & $47(3)$ & $59(3)$ & $51(3)$ & $2(2)$ & $2(2)$ & $-6(2)$ \\
\hline$C(2)$ & $46(4)$ & $75(4)$ & $52(3)$ & $7(3)$ & $7(3)$ & $-1(3)$ \\
\hline$C\left(2^{\prime}\right)$ & $30(3)$ & $57(3)$ & $49(3)$ & $3(2)$ & $1(2)$ & $-4(2)$ \\
\hline$C(3)$ & $58(3)$ & $50(3)$ & $52(3)$ & $0(3)$ & $3(3)$ & $-8(3)$ \\
\hline$C(4)$ & $135(7)$ & $90(5)$ & $97(6)$ & $36(4)$ & $35(4)$ & $-6(5)$ \\
\hline$C(5)$ & $46(3)$ & $61(3)$ & $46(3)$ & $-5(2)$ & $-3(2)$ & $5(3)$ \\
\hline$C(6)$ & $82(4)$ & $48(3)$ & $77(4)$ & $8(3)$ & $-16(4)$ & $-9(3)$ \\
\hline$C(7)$ & $138(7)$ & $93(5)$ & $76(5)$ & $-9(4)$ & $-20(4)$ & $-46(5)$ \\
\hline$C(8)$ & $122(6)$ & $114(6)$ & $66(4)$ & $15(4)$ & $-8(4)$ & $-24(5)$ \\
\hline$C(9)$ & $132(7)$ & $70(5)$ & $194(11)$ & $22(5)$ & $-25(7)$ & $12(5)$ \\
\hline$C(10)$ & $98(5)$ & $104(5)$ & $68(4)$ & $14(4)$ & $9(4)$ & $14(4)$ \\
\hline$C(11)$ & $149(7)$ & $96(5)$ & $98(6)$ & $6(5)$ & $10(5)$ & $13(5)$ \\
\hline$C(12)$ & $87(5)$ & $155(7)$ & $100(6)$ & $-54(5)$ & $15(5)$ & $-25(5)$ \\
\hline
\end{tabular}

